\title{
PEDAGOGÍA, AUTOCONOCIMIENTO Y GESTOS DESCOLONIALES EN LAS ALTERNATIVAS A LA EDUCACIÓN FORMAL
}

\author{
FRANCISCO RAMALLO
}

Doutor em Humanidades e Artes com especialização em Ciências da Educação (Universidade

Nacional de Rosário). Professor e pesquisador do Departamento de Ciências da Educação, Faculdade de Letras, Universidade Nacional de Mar del Plata (UNMdP-CONICET), Argentina, onde trabalha como assistente de ensino da cadeira Problemática Educação. Membro do Grupo de Pesquisa em Educação e Estudos Culturais (GIEEC) e a Rede Tejidos - Pedagogias do Ser:

Educadores, Experiências Descoloniais e Queers - ambos baseados no Centro de Pesquisa Multidisciplinar em Educação (CIMED). ORCID 0000-0002-4611-3988. E-mail: franarg@, hotmail.com 


\section{PEDAGOGÍA, AUTOCONOCIMIENTO Y GESTOS DESCOLONIALES EN LAS ALTERNATIVAS A LA EDUCACIÓN FORMAL}

En las alternativas a la educación formal el autoconocimiento -aquello que está en el interior de cada sujeto- ocupa recurrentemente un lugar primordial. En este artículo a partir de una serie de relatos de experiencias de educación no formal en Mar del Plata (Argentina) -particularmente alternativa, como sulen autorefereniciarse-, reconocemos un pedagogía de y para ser compuesta por gestos descoloniales. En un primer momento exploramos una posible genealogía entre la pedagogía, el autoconocimiento y la descolonialidad del ser. Para luego relfexionar sobre los gestos descoloniales que convergen en tres experiencias en las que desde interior del sujeto y lo desconocido se componen otros mundos (posibles).

Palabras claves: Educación no formal, Narrativa, Pedagogía, Pensamiento descolonial.

\section{PEDAGOGY, SELF-KNOWLEDGE AND DECOLONIAL GESTURES IN THE ALTERNATIVES TO FORMAL EDUCATION}

In the alternatives to formal education, both self-knowledge and what is within each subject usually take an important role. In this article, and taking into account a series of narratives about non-formal- or alternative, as it is usually self-addressed- education in Mar del Plata (Argentina), we recognize a pedagogy of and to be composed by decolonial gestures. Firstly, we explore a possible genealogy among pedagogy, self-knowledge and decoloniality of being. Later, we reflect upon decolonial gestures which converge in three experiences where other (possible) worlds are composed from within the subject and the unknown.

Keywords: non-formal education, narrative, pedagogy, decolonial thought.

\section{PEDAGOGIA, AUTOCONHECIMENTO E GESTOS DECOLONIAIS NAS ALTERNATIVAS À EDUCAÇÃO FORMAL}

Nas alternativas à educação formal, o autoconhecimento -aquilo que está dentro de cada sujeito - geralmente ocupam um lugar primordial. Neste artigo, baseado em uma série de relatos de experiências de educação não formal em Mar del Plata (Argentina) -particularmente alternativa, como sulen autorefereniciarse-, reconhecemos uma pedagogia de e para ser composta de gestos descoloniais. Inicialmente, exploramos uma possível genealogia entre pedagogia, autoconhecimento e a descolonialidade do ser. Para os efeitos de refletir sobre os gestos descoloniais que convergem em três experiências nas quais outros (possíveis) mundos são compostos desde dentro do sujeito e desde o desconhecido.

Palabras clave: Educação não-formal, Narrativa, Pedagogia, Pensamento descolonial. 


\title{
PEDAGOGÍA, AUTOCONOCIMIENTO Y GESTOS DESCOLONIALES EN LAS ALTERNATIVAS A LA EDUCACIÓN FORMAL
}

\author{
Introdução
}

Lo que le niegues a los demás también te será negado, por la simple razón de que estás siempre legislando para ti mismo: todas tus palabras y acciones definen el mundo en que deseas vivir. Thaddeus Golas (2006, p. 44).

En las propuestas de enseñanza que componen las alternativas a la educación formal recurrentemente el denominado autoconocimiento suele ocupar un lugar protagónico. Entendido como la propia capacidad de conocer aquello que está en el interior de cada sujeto interpela un despertar, que -en palabras de una libre educadora- alude a "una toma de consciencia de sí para vivir en armonía con nosotros mismos y con el todo que nos rodea y del cual formamos parte" (Entrevista 3) ${ }^{1}$. En tanto podríamos destacar que las pedagogías que sedimentan las alternativas a la educación formal suelen guardar una estrecha relación con examinar quiénes somos, como un cuestionar de nuestra visión del mundo (o los mundos) que reconocemos y del lugar que ocupamos en él (o en ellos) (RAMALLO, 2018).

En la invención de pedagogías alternativas que recuperan una dimensión ontológica, fundada y fundante del autoconocimiento-nombrado de esta forma a partir de las voces vinculadas a las experiencias aquí registradas - se propone inicialmente ser uno mismo y no intentar convertirse en nada que uno ya no sea. Así como la celebración de estar en contacto con otras existencias y el hecho de apreciar la plenitud de cada momento en el que estamos presentes. El conectarse con el todo que componemos y que nos rodean proporciona una visión complementaria a la predominantemente reduccionista y materialista imperante en el pensamiento $\mathrm{y}$ en las instituciones educativas formales -capaces de ser reconocidas como occidentales, modernas, coloniales, eurocéntricas-. En la pedagogía esta visión totalista encuentra ecos tanto en grandes movimientos sociales como en pequeñas experiencias educativas que recuperan herencias ancestrales que no son ni particularmente místicas, ni índigenas - o nativas americanas-

\footnotetext{
${ }^{1}$ La escritura de este trabajo se compone a partir de las observaciones realizadas por el autor y las entrevistas a diferentes actores vinculados a la experiencias de educación no formal -alternativa- en Mar del Plata.
} 
ni africanas ni orientales ni de cualquier otra índole identitaria geo-cultural determinada. Sino que se nutre de voces, textos y experiencias diferentes, manchadas, híbridas, mestizas y alternativas a la forma de educar occidental consagrada. Entre la dialéctica de la inclusión y la crítica, las cosmologías interrumpidas de los pueblos no occidentales - o colonizados -, los aportes de los pensadores místicos sufis o hinduistas en sus combates a la occidentalización, el budismo y los diferentes saberes de las religiones del mundo, la ancestralidad y la intimidad con lo legado, Steiner y la antroposofía filosófica o la New Age -aunque tan lucrativa y ligada a la expansión de una cultura comercial- constituyen una apertura a poder imaginar la educación de otra forma. Además de ofrecer prácticas transformadoras, que cambian a un mismo tiempo el yo y el mundo (o los mundos) que componemos y descomponemos.

Estos desplazamientos se pueden comprender en los nuevos tiempos del siglo XXI, en el que cada vez se visualiza aún más como el explorar el ser interior constituye un recurso poderoso para la movilización social y para el pensamiento crítico en la educación. En palabras del pedagogo del norte Phipil Wexler (2008) el siglo XXI es portador de lenguajes y posibilidades que alimentan una "resacralización de la educación", como un movimiento que desde diferentes contextos educativos intenta ir más allá de lo que conocemos en términos de lo real, recogiendo lo sagrado de la existencia humana en oposición a una suposición racionalista. Esta consciencia surgió, a decir de Wexler (2008), de reconocer que con la racionalización del discurso científico se abandonó el poder de lo mágico y lo sagrado. Y que sin ese poder los aspectos racionales de la educación parecieran olvidar sus más profundos sentidos.

Además entre sus más amplias concepciones y evitando cualquier intento de delimitar un vasto campo tan difuso como desconocido, las pedagogías del autoconocimiento se asocian a los paradigmas libertarios en la educación. Especialmente es a partir de los años ochenta y noventa del siglo XX y sobre todo en el nuevo siglo cuando comenzaron a circular textos y experiencias de las pedagogías en la búsqueda de la libertad del ser y la invención de una educación antiautoritaria. Quizás la voz de Rebeca Wild (1996) a partir de su labor en la Escuela Experimental Pestalozzi, sea aquí la referencia más profunda y representativa.

De manera tal que estas pedagogías están asociadas tanto a grandes movimientos culturales, sociales y políticos como a pequeñas (en algunos casos muy pequeñas) experiencias de autogestión educativa. En este ejercicio de indentificación, si tuviéramos que recuperar algunas de sus tramas, podríamos decir que las pedagogías del autoconocimiento se asocian a la invención de propuestas de aprendizaje y de enseñanza que se caracterizan por la convivencia, la autonomía y la participación quíenes se educan en la toma de decisiones. Al respecto Cuevas Noa (2003) presentó tres aspectos que componen lo común en las pedagogías libertarias, que 
cobran sentido en esta conversación. El primer rasgo que las define es el antiautoritarismo, a partir de evitar la sumisión y proponer aprender de la autonomía y de la libertad. A la vez que la educación se proyecta no desde la autoridad del maestro sino desde los intereses de cada uno de sus estudiantes, intentando conseguir que sean dueños de sus propias vidas. Ello implica la libertad de su autonomía frente a la opresión y explotación que la educación tradicional provoca. En segundo término, la autogestión es un rasgo de estas pedagogías, siendo el control de la educación responsabilidad de cada individuo. La autogestión supone varios aspectos, desde la capacidad de construir espacios educativos y la autorganización de los estudios por parte del grupo hasta la autogestión de los aprendizajes y el autodidactismo. Y finalmente es la educación integral para Cuevas Nova (2003), otro rasgo común de las pedagogías libertarias que alude a la completud y no fragmentar o dividir la totalidad de la educación humana. En los términos de este pedagogo español se refieren a educar para la vida social en un sentido holístico, por ejemplo sin separar el trabajo manual del trabajo intelectual. Aquí remarcamos además al autoconocimiento y el derecho a ser que se implica en el educar(nos) como uno de los rasgos constitutivos en estas difusas y emergentes pedagogías.

\section{La des-colonialidad del ser en la educación no formal}

En las alternativas a los modos formales de educar que registramos en el proyecto de investigación "Formas de Educación alternativas en el siglo XXI", notamos que explorar el ser es además una irrupución que se propone frente a la denuncia de la "colonización pedagógica" que constituye a la escuela tradicional ${ }^{2}$. En efecto, la "otra educación" que se ofrece desde los caminos desandados por experiencias no formales -o alternativas- de educación y por los discursos y las prácticas de la descolonialidad se funda en otro modo de $\operatorname{ser}^{3}$. Lo que según Catherine Walsh podría ser interpretado como "maneras distintas de ser, pensar, conocer, sentir, percibir, hacer y vivir que desafían la hegemonía y universalidad del capitalismo, la modernidad eurocéntrica y la lógica civilizatoria occidental (WALSH, 2014, p. 20).

En estos recorridos la des-colonialidad del ser se presenta como un aspecto central que caracteriza a las "grietas" que recuerdan los vicios más profundos de lo que llamamos, en la modernidad eurocentrada, "educación" (WALSH, 2014). Por lo que se vuelve importante interpelar a la educación interior desde una lectura de los alcances y los límites de la categoría

\footnotetext{
${ }^{2}$ Proyecto de Investigación desarrollado entre 2016 y 2017 radicado en el Grupo de Investigadores en Educación y Estudios Culturales (GIEEC) de la Facultad de Humanidades, UNMdPta.

${ }^{3}$ El uso de la "des"-cons-alude a una mirada respetuosa y a la vez irreverente respecto del programa académico-político modernidad/colonialidad. En el sentido que autoarroga una potestad para nombrar a partir de nuestros lenguajes y enunciaciones biográficas y geo-culturales.
}

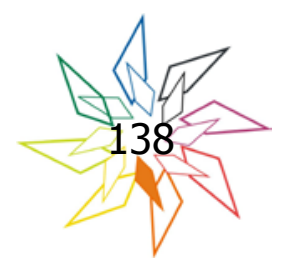


"colonialidad del ser". Como así también de las pertubaciones que se registraron a partir de la reflexión y la observación de algunas de las experiencias de educación no formal que analizamos en nuestro proyecto de investigación ${ }^{4}$. Recordamos que dentro de la emergencia del denominado pensamiento descolonial en la academia, algunos estudiosos y activistas destacaron una serie de dimensiones que afectan nuestras existencias cotidianas (la colonialidad del poder, del saber, del ser y de la naturaleza). Estas categorías conceptuales se asocian a la búsqueda de nuevas perspectivas geopolíticas del conocimiento no eurocéntricas y a un conjunto amplio de relatos caracterizados por la "diferencia colonial" y el "vuelco de la razón" (MIGNOLO, 2011). En una mirada que refiere al cuestionamiento de la centralidad epistemológica del ser y del poder europeo-norteamericano y que recupera en su mismo desarrollo lo marginalizado e invisibilizado de nuestras memorias ${ }^{5}$.

Específicamente el concepto "colonialidad del ser" surgió en relación a la reflexión del racismo y la experiencia colonial. A partir de la rearticulación de las preguntas sobre el ser de Martin Heidegger, Jacques Derrida, Emamanuel Levinas, Rudolf Steiner y un poco más acá Frantz Fanon y Enrique Dussel. Este último filósofo de la liberación notó la conexión entre el ser y las empresas coloniales y fue Walter Mignolo quien formuló el concepto en el marco de las conversaciones de la colonialidad del poder. Para Mignolo (2011) esta dimensión de la colonialidad se refiere a la experiencia vivida de la colonización y su impacto en el lenguaje, en la capacidad de expresar lo que uno es o cree ser. Además, dentro de un programa de estudio y de intervención política de las ciencias sociales en América, Nelson Maldonado Torres fue quién mayor atención le prestó a esta dimensión, resaltando los efectos de la colonialidad en la experiencia vivida cotidiana y no sólo en la mente de sujetos subalternizados (Maldonado Torres, 2007).

Anteriormente fue Franz Fanon quién colocó la pregunta donde no había sido nunca antes colocada, interrogándose existencialmente sobre la posibilidad de ser negro en el mundo moderno y colonial. En su libro Piel negra, máscaras blancas (1973) describió la emergencia de un esquema

\footnotetext{
${ }^{4}$ Los registros de experiencias son compuestos a partir de una mirada biográfico-narrativa institucional alimentanda por los aportes de la investigación narrativa en educación (Yedaide, Álvarez y Porta, 2015), que la comprende no sólo en términos metodológicos sino también conceptuales como un modo otro de conocer. ${ }^{3} \mathrm{El}$ uso de la "des"-cons-alude a una mirada respetuosa y a la vez irreverente respecto del programa académico-político modernidad/colonialidad. En el sentido que autoarroga una potestad para nombrar a partir de nuestros lenguajes y enunciaciones biográficas y geo-culturales.

${ }^{5}$ Esta propuesta intelectual pone en evidencia las articulaciones, estrategias, mecanismos y categorías propias del eurocentrismo; como la raza, el control del trabajo, el Estado, la escuela y la producción de conocimiento. Afirmadas en la modernidad como patrón civilizatorio, éstas irrumpen y permean los diferentes ámbitos de la vida humana, a partir de la dominación, el control y la explotación sistemática del ser, así como en el ocultamiento del otro (el "otro" es el diferente al europeo/occidental) y de sus posibilidades de realización y de reconocimiento histórico y existencial
} 
histórico-racial que traumatizó la subjetividad del negro y lo convirtió en una excepción de lo humano, ya que lo tiene atrapado en un no-lugar dado que las concepciones típicas sobre lo que es humano no son aplicables a su existencia. En tanto delimitó una "zona del ser" (mundo blanco) y una "zona del no ser" (mundo negro), pues el negro tiene un solo destino: emblanquecer o morir. En esta obra iniciática del pensamiento descolonial, la reflexión sobre la línea de color aplicada a los humanos expresó los límites de la educación moderna (eurocéntrica y occidental) de los pueblos colonizados. Interpelando a los colonizados a desaprender la racionalización del pensamiento occidental y fundando una pedagogía del ser y una educación para existir (RAMALLO, 2015).

La pedagoga ecuatoriana Catherine Walsh (2015) reconoció la posibilidad de in-surgir, re-existir y re-vivir, a pesar de la (re)colonización de los pueblos de la región, desaprendiendo la educación moderna en proyectos que apunten a la re-existencia y a la vida misma. A partir de un imaginario otro de con-vivencia (de vivir "con") en una pedagogía de ser y de hacerse humano, que tal como recuperó con la voz de Stephan Haymes se trata de una educación construida y por construir que incluye la topología del ser y la teología identitaria-existencial de la diferencia colonial (Walsh, 2015; 15).

Si bien desde este recorrido conceptual, existencial y experiencial se advierte la dolorosa "colonialidad de ser", las experiencias que alimentaron la escritura de este texto dejaron ver que esta categoría, instrumento político del programa que propone Walsh (2015), suele ser interpretada desde una dimensión que se podría denominar como externa al ser. En tal sentido preferimos comprender una definición de esta dimensión de la colonialidad a partir del acto existencial de descolonizar al ser interior, insistiendo en un despertar propio como una energía desconocida que ya existe. Y más aún, que por su propia definición anula la posibilidad de delimitarse, aunque no obstante exista y se invoca cuando se desaprenden los actos de la educación exteriorizadora, moderna y civilizadora. Por tanto entedemos que la descolonialidad del ser como categoría heurística posibilita registrar las experiencias en la educación que componen una dimensión interior del sujeto y marcada por lo que vive y cree vivir cada sujeto.

Más allá de lo señalado creemos que lo que podría pensarse como la descolonialidad del ser es entramado desde recorridos posibles fundados en las experiencias de una educación libre, no directiva y fuera de la gramática de la escuela. Pues notamos que muchas veces en ellas emerge un espíritu posible de comprenderse como descolonial. Aunque por supuesto no de manera lineal y esencialista, en el sentido de que no todas las experiencias no formales son factibles de ser interpretadas en el terreno de la búsqueda y la vida descolonial.

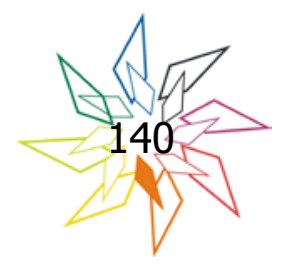


Aquí vale la pena recordar que con notable profundidad Esteva, Prakash y Stuchil (2013) relfexionaron sobre cómo la educación formal implica querer "concientizar a un otro", insistiendo en la necesidad de revisar la idea de lo que llamamos "educación" en la modernidad. Además de realizar una contundente crítica a la idea de mediación -que a partir de la "concientización" anula las capacidades de ser- desde un llamamiento a liberar la pedagogía. De hecho consideraron que el propio Freire, maestro fundante de la pedagogía crítica, proponía un cambio que no comienza por y desde el sujeto colonizado sino que parte de una concientización externa. Convencido de que tanto opresores como oprimidos son deshumanizados por la opresión, esta visión suponía que una nueva conciencia nacida del alfabetismo ilustrado permitiría hacerse de nuevo plenamente humano y a la vez eliminar los horrores de la opresión moderna (Esteva, Prakash y Stuchil, $2013 ; 34)$. También explicaron estos autores que ello podría comprender como una arrogancia de quíenes se colocan en una clase superior. De modo que Freire se ubicó a sí mismo en una tradición que descarta, suprime o descalifica -implícita o explícitamente- la abundante evidencia histórica de que los sujetos se rebelan por sí mismos contra toda clase de opresores. En una operación que implica la arrogancia insostenible de poseer la conciencia "verdadera" (o universal) y el propósito de legitimar el derecho de intervención en las vidas de los demás.

Es por ello que esta actitud de mediación de la educación moderna, con la intención de modelar y no orientada al ser, constituye una ceguera de los educadores en relación a su propia participación en la opresión de aquellos a los que se pretende concientizar. Al presumir que han logrado alcanzar un nivel avanzado de madurez, conciencia o incluso liberación, de veda una concienciatización interna. En contraposición a ello la "pedagogía no directiva" que propuso Wild (1996) remarcó la necesidad de educar para ser. A partir de la reflexión sobre la construcción de un espacio alternativo de enseñanza en Ecuador, esta pedagoga insitió en la necesidad de educar desde la vida en la libertad; en un vivir la vida y de la educación de un modo propio y personal. Fundada en el rechazo del autoritarismo -pero no en la autoridad en sí misma- propone un educador que aprenda a respetar las estructuras mentales y emocionales del educado. En un ambiente en el que los educadores y los educados, con dudas y curiosidades, crezcan seguros de sí mismos y sobre todo en la capacidad de autoeducarse. Una educación para ser, en estos términos, está orientada hacia la experiencia del momento, la necesidad de estar en permanente movimiento y en permanente expansión de lo que nos autoconstituye.

Asimismo ello implica una educación para dejar al educado tomar su propio camino, o mejor dicho dejar simplemente ser. Olvida cualquier pretensión autoritaria de concientización externa y provoca un desprendimiento de la premisa de la escuela como lugar de realización del sujeto, en el sentido que uno crea, sueña e imagina otras posibilidades fundadas en lo que somos. En diferentes experiencias que se escapan a la lógica de la escuela tradicional, desde 
diversos posicionamientos y modos de educar, la educación del ser se presenta como el punto de partida primordial. Pese a la ambigüedad y variabilidad de sentidos de esta premisa, lo alternativo muchas veces parecería estar construido por una idea propia de ser. Una idea que a diferencia de la educación hegemónica e instituida es libre y con sentido propio.

Las experiencias observadas en nuestro proyecto de investigación en su mayoría están conformadas por grupos de trabajo reducidos, con un fuerte vínculo con la naturaleza y con una fuerte apuesta a reemplazar los valores de la competencia por valores colaborativos, que proponen una educación del ser, que es y que se hace. Tal como expresó la educadora de una de las experiencias educativas visitadas: "la educación es, la educación sucede y es parte de nuestras vidas desde que llegamos a este mundo y hasta que nos retiramos de él (Entrevista 1).

En Tierra Fértil, una escuela de educación libre, por ejemplo se plantea el principio de que el niño pueda apropiarse libremente del ambiente, de manera que todo debe estar a su alcance, para que pueda orientarse y moverse con autonomía. Los niños allí son libres de salir y entrar de las actividades cuando lo necesitan según sus necesidades y deseos. Se considera que el juego libre permite aprender a trabajar en colaboración, establecer reglas consensuadas y resolver los conflictos de un modo pacífico y auto-regulado. Las guías organizan los ambientes a partir de propuestas lúdicas y didácticas que tienen en cuenta el proceso evolutivo en el que se encuentran y las necesidades individuales de cada niño. Se busca la expansión interior, a partir de que lo experimental, lo intuitivo y lo espontáneo se encuentran con lo comunitario, lo emotivo y lo espiritual. Ello conforma un ecléctico escenario pedagógico en el que hasta la alimentación y el cuidado del mundo interior constituyen una otredad con respecto a la gramática escolar (entrevista 1$)^{6}$.

En el mismo sentido EnRonda, una escuela alternativa que funcionó entre el 2013 y el 2016 en el Barrio El Alfar de Mar del Plata, a través de un cambio en la forma de educar se pretendía despertar una conciencia individual y social para crear un mundo mejor, a partir de "dejar ser libremente a los niños" (entrevista 2). Un mundo basado en la solidaridad, el respeto por la diversidad, la armonía en la convivencia, el cuidado de sí mismo, del otro y del entorno, la empatía, la escucha mutua y la autorregulación. Lo que pretendía ofrecer EnRonda era un acompañamiento personalizado y un ambiente preparado para que los chicos pudieran desarrollar su potencial

\footnotetext{
${ }^{5}$ Tierra Fértil surgió en 2014 a partir de un grupo de familias que se propuso para sus hijos una educación libre y no directiva. Este proyecto no se define como una escuela sino que se plantea como un ámbito educativo que tiene como ejes transversales la autogestión, la autoeducación, la autonomía, la democracia, la libertad y el juego libre.
}

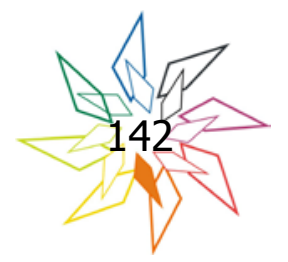


interno, cada uno a su propio ritmo, a partir de su curiosidad y su deseo de jugar y de aprender (entrevista 2). Una marca que aquí aparece es la autoexploración del cuerpo como una manera de conocerse y registrar aquellos que olvida la educación formal. En ese autoconocimiento interior, que Fanon (1973) rememoró como vedado en la escuela moderna, se componen actividades que provocan una escucha hacia uno mismo y el estar disponible para integrar las necesidades del organismo humano a partir de una toma de conciencia sensorial y vital?

Por su parte en El Canto del Fuego se propone "una educación que puede ser de otra manera que la que existe en este momento, más amorosa y respetuosa del ser" (entrevista 3$)^{8}$. Este espacio educativo busca generar un contexto para que el aprendizaje surja, más que dirigir el aprendizaje. Se compone como "un ambiente que permite la creatividad y el autoconocimiento en los niños, acompañados por adultos responsables que los guíen en su camino parar generar su propio aprendizaje -impulsados por sus necesidades internas-" (entrevista 3). Este proyecto es guiado de manera grupal entre las educadoras y las familias, "en el que nada está dicho, cada día se van probando nuevas ideas y nuevas maneras de educar" (entrevista 3). Lo que implementan es lo que van viviendo cada día y la educación que se proponen es emocional y espóntanea. Diría nuestra informante "todo el tiempo estamos atentas a lo que van viviendo los chicos y también a lo que vamos viviendo nosotras, por eso el trabajo con uno mismo es muy grande y además todo lo tenemos que ir creando" (entrevista 3).

Las tres experiencias aquí brevemente expuestas si bien se tratan de espacios de aprendizajes para niños, también se proponen contribuir a que los adultos -tanto del grupo que lo componen como de la comunidad en general de la que forman parte- se autoeduquen en el respeto de las necesidades de los seres humanos. Estas experiencias alimentan pedagogías indisociables de la totalidad de la experiencia humana, que se alimentan tanto de cosmologías interrumpidas como de saberes cotidianos que comúnmente despreciamos. En lo que se aprendió para vivir en armonía con nuestro ser, con lo que somos y con lo que nos contacta con la creación humana, estas pedagogías vivas aprenden en la libertad de reconocer(se) y tomar conciencia de quién se es.

\footnotetext{
${ }^{7}$ EnRonda inició su experiencia en 2013 en el barrio El Alfar de Mar del Plata. Luego de organizar varias reuniones un grupo de educadores y de padres abrió un espacio autoproclamado como educación libre.

${ }^{8}$ El Canto del Fuego es una escuela de educación libre y consciente iniciada en 2015, que funciona en la zona céntrica de la Ciudad de Mar del Plata.
} 


\section{Gestos descoloniales en otros mundos (posibles)}

En este breve texto exploramos los relatos de una pedagogía para permitir(nos) inventar, experimentar y crear a partir del autoconocimiento y de dejar en libertad el deseo de aprender y de crecer que todos tenemos dentro. Estas marcas enuncian que la posibilidad de crear un espacio para ser, es componer un espacio para nuestra propia descolonización y viceversa. Dar un paso al costado y corrernos de la colonización pedagógica, en las voces de las experiencias aquí registradas, parecería ser algo así como conectarse (o reconocerse) con uno mismo, con la esencia de lo que uno mismo es y lo que cada uno posee dentro de sí. A partir de una des-colonialidad del ser estas pedagogías proponen liberarse de lo impuesto y de lo ajeno. El autoconocimiento en la educación implica discriminar dentro y con uno mismo qué es lo que somos, lo que queremos y lo que deseamos (y qué es lo que no somos, lo que no queremos y lo que no deseamos). Es preguntar por nuestros deseos y no por los deseos de los otros sobre nosotros.

En ese sentido es que se rechaza la colonización -lo exterior, lo impuesto y lo ajenointerpelando un andar inventivo por lo no dado, a partir de un recorrido desconocido y experimental guíado por nuestro interior. Diría una de las educadora de estas esperiencias que lo posible está dentro de nosotros, "ya que si lo puedes sentir o pensar lo puedes crear" (entrevista 3). Es la invención una propuesta de "resetearse" y de interrogar qué somos y qué podemos llegar a ser, rechazando lo impuesto y lo que no somos, para reconocerse y reconectarnos con identidades intímas y memorias profundas. Una educación para el autoconocimiento, entonces, sería facilitar, experimentar, inventar y crear un espacio para que uno mismo pueda conectarse con sus deseos y evitar recibir los mandatos externos que nos domestican y nos colonizan. Se trata también de que uno pueda conocer sus deseos en la educación, indagando su propio desarrollo personal y su íntimo deseo de autorrealización en relación con el o los mundos que nos constituyen.

Más aún, la idea de educación más allá de lo conocido implica fundamentalmente buscar algunas respuestas en lo más íntimo que nos compone. Ser en la educación también es recordar que la forma de educar tradicional (mayormente aceptada y compartida propia de la escuela) es una entre otras posibles. La educación es invención y si bien en la imposición de la modernidad eurocentrada el aprendizaje escolar fue redefinido como educación, siempre existieron otras formas y otros espacios que constituyen una genealogía de otros modos de educar. Especialmente en los últimos años emergieron experiencias de educación alternativa que muchas veces (aunque no necesariamente siempre) se escapan y se desprenden de la educación escolar tradicional a partir de proponer un apertura y hacia nuevos espacios para la libertad humana en la educación.

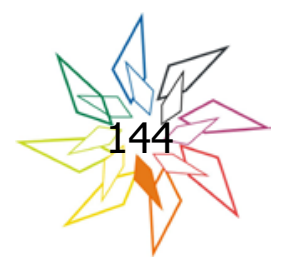


Si bien la colonialidad sea más fácilmente advertida en todas las formas de explotación del trabajo y del control de la producción-apropiación-distribución material, es la colonialidad del ser, esa "colonialidad interior", la que marca profundamente nuestro pensar, sentir y actuar cotidiano. En todo aquello que forma parte de nuestra subjetividad, como conductas que marcan inquietudes en todo tipo de diferencias raciales, de sexo, de género, de participación en la vida del trabajo, etc. Y es la escuela tradicional, como institución moderna por excelencia, la que se encargó de reproducir, desde sus inicios hasta la actualidad, aquellos ideales de razón, progreso y capital como horizontes de realización del sujeto moderno. Desde esta perspectiva la escuela moderna confunde enseñanza con saber, promoción con educación, diploma con competencia y fluidez con la capacidad para decir algo nuevo. Se "escolariza" la imaginación del alumno, diría Ivan Illich. Todos los esfuerzos se depositan en el acrecentamiento de la producción y no en la formación de personas que vivan una vida plena y contribuyan a que también otros logren la plenitud.

Es por esto que una pedagogía del ser se proyecta en la búsqueda de aquella plena humanidad que voces como la de Fanon (1973), y otras más anónimas, tanto nos enseñaron a ambicionar e imaginar. Entonces sería oportuno abandonar las formas de conocer que sujetan y modelan activamente nuestras subjetividades en las fantasías de las ficciones de la modernidad. Aquellas subjetividades modernas devotas del consumo, de la idea de que la economía es la ciencia de lo existente, de las concepciones patriarcales y racistas, para pasar a concebir espacios de sociabilidad, intercambio y encuentro, viviendo en comunidades donde se trabaje para vivir y no solo se viva para trabajar y consumir. Estos serían caminos que celebren la descolonización del ser, entramados desde otros recorridos posibles, fundados en las experiencias de una educación libre, no directiva y fuera de la gramática de la escuela en la que muchas veces emerge interiormente un espíritu descolonial.

Esta educación desde el autoconocimiento camina por pequeñas grietas que provocan vertiginosas y profundas transformaciones en el modo de pensar/sentir/hacer el territorio de la educación y que implican consolidar posicionamientos más allá y más acá de las ideas y las prácticas del doloroso proyecto universal de la modernidad (Walsh, 2014). En pequeños quiebres que a pesar de su fragilidad, incertidumbre, inseguridad, escurridez y riesgo, lejos de ser poco importantes convocan a habitar una pedagogía descolonial no excluyente y situada en la inmediatez de nuestros gestos (Porta y Yedaide, 2017). En el sentido de volver la mirada al escrutinio de las formas en que el contexto devela gramáticas del sufrimiento enlazadas al legado eurocéntrico en nuestro entorno social y educativo. En esa inmediatez reconocemos que una pedagogía descolonial espaca de lo normativo, recetario y directivo en pos de pequeños gestos en grietas de libertad. 
Entonces abrir una dimensión desde y con el ser en la educación es abrir posibilidades, en una invención que alimenta otras maneras de educar, desconocidas y posibles de ser imaginadas. $\mathrm{Y}$ en ese reconocer ya no habitamos un mundo, sino que son las grietas las que lo quiebran y nos desvían a confiar en la composición de otros mundos posibles. El educarnos conociéndonos a nosotros mismos, es también reconocer que ya no hay un solo mundo, sino que son muchos los posibles. Si bien en el filosofar contemporáneo no existe un acuerdo sobre qué son los mundos posibles (y por supuesto no tendría por qué existir), esta metáfora, y reapropiado lema político que recorre desde hace décadas nuestro continente, devela el riesgo, la discontinuidad y la aventura de soñar con lo imaginado.

Estos gestos descoloniales en las alternativas a la educación formal componen otros mundos posibles. Vale la pena recordar aquí que en la filosofía y la lógica occidental la noción de mundos posibles suele aludir a una noción de conjuntos de proposiciones maximales (es decir que se les puede agregar cualquier otra proposición), como entidades abstractas y si se quiere platónicas. De hecho, según el realismo modal de David Lewis, los mundos posibles son universos, y nuestro universo es sólo uno entre muchos. Para otros los mundos posibles no son algo que se descubre, sino que se estipulan mediante descripciones imaginadas. Pero además la multiplicidad de los mundos en los que vivimos también se presentó en el pensamiento pedagógico contemporáneo y quizás el educador austríaco Rudolf Steiner fue el que más visible lo hizo en relación a los "mundos superiores"; aquellos que asoció justamente a la conciencia espiritual y al sendero de conocimiento de sí que conduce de lo espiritual en el hombre a lo espiritual en el universo (uno de los puntos fundantes de la antroposofía). Y con más fuerza fue la irrupción descolonial, entramada en el ser, a partir de la construcción de saberes tanto fuera como dentro de los parámetros de la ciencia y la filosofía occidental, la que introdujo la metáfora de los otros mundos posibles en el pensamiento pedagógico. En tanto los saberes "otros", el vuelco de la razón, los desprendimientos del pensamiento colonial y la ruptura con la celebración de la modernidad, colocaron a la existencia de otros mundos posibles como un horizonte para imaginar(nos).

A partir de las conexiones entre las pedagogías del autoconocimiento, las grietas descoloniales y los otros mundos posibles, en nuestra inmediatez se propone un tiempo de imaginar, crear, inventar y soñar mucho más alto de lo que lo hasta ahora estuvimos haciendo. El abandono del temor de dejar entrar al ser interior en la educación, las experiencias de invención de otras maneras propias de educar libres y autogestionadas, la exploración de uno mismo y el autoconocernos como ideal educativo colocan grandes y desesperantes interrogantes en los sentidos de educar en el siglo XXI, a la vez que nos prometen una cierta tranquilidad al imaginar que las respuestas están dentro de nosotros mismos.

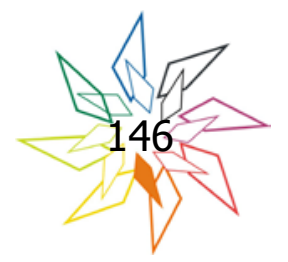




\section{REFERÊNCIAS}

CUEVAS NOA, F. J. Anarquismo y educación: La propesta sociopoLitica de La pedagogia Libertaria. Madrid: Fundación de Estudios Libertarios Anselmo Lorenzo, 2003.

ESTEVA G., PRAKASH M. Y STUCHUL D. De la pedagogía de la liberación a la liberación de la pedagogía. En: Aprendizaje en movimiento. Rutas hacia la liberación de la Pedagogía. México: Palpa Editorial, 2005.

FANON, F. Piel Negra, Máscaras Blancas. Buenos Aires: Editoral Abraxas, 1973.

GOLAS, T. Manual de Iluminación para holgazanes. Buenos Aires: Del Nuevo Extremo, 2006.

MALDONADO TORRES, N. Sobre la colonialidad del ser: contribuciones al desarrollo de un concepto En: Castro Gómez, S. y Grosfoguel, R. El giro decolonial: Reflexiones para una diversidad epistémica más allá del capitalism global. Bogotá: Siglo del Hombre Editores, 2007.

MIGNOLO, W. El vuelco de la razón. Diferencia colonial y pensamiento fronterizo. Buenos Aires: Ediciones del signo, 2011.

PORTA, L. y YEDAIDE, MM. (2017) Pedagogía(s) vital(es): Cartografías del pensamiento y gestos ético-políticos en perspectiva descolonial. Mar del Plata, EUDEM, 2017.

RAMALLO, F. Pedagogías, descolonización y mundo negro: Una convergencia necesaria en la búsqueda de los otros mundos posibles. En: Fernandes, M y Meireles, M (coordinadoras) Educação, Diversidade e Diferenças: Olhares (des)colonizados e territorialidades múltiplas. Curitiba: CVE, 2015.

RAMALLO, F. El conocimiento de sí y las pedagogías del ser: Un relato de la experiencia de la escuela El canto del Fuego. Revista Cronía Año 18 - No 14, 2018.

SEGATO, R. La crítica de la colonialidad en ocho ensayos. Y una antropología por demanda. Buenos Aires: Prometeo Libros, 2015.

WALSH, C. Pedagogías decoloniales caminando y preguntando: notas a Paulo Freire desde Abya Yala. Revista Entramados. Educación y Sociedad. Nº1, Año 1, 2014. 
WALSH, C. Pedagogías decoloniales: Prácticas insurgentes de resistir, (re) existir y (re) vivir. Quito: Serie Pensamiento decolonial, 2015.

WEXLER, P. La religión como crítica socioeducativa: Un ejemplo weberiano”. En: McLaren, Peter -Kincheloe, Joe (eds.). Pedagogía crítica: De qué hablamos, dónde estamos. Barcelona: Grao, 2008.

WILD, R. Educar para ser. Vivencias de una escuela activa. Buenos Aires: La Chamaquería, 1996.

YEDAIDE, MM. ÁLVAREZ , Z y PORTA, L. La investigación narrativa como moción epistémico-política. Revista Científica Guillermo de Ockham Número 13 Volumen 1, 2015.

\section{ENTREVISTAS}

Entrevista 1. Malala, educadora responsable de Tierra Fértil. Entrevistada por Francisco Ramallo en abril 2016. Proyecto de Investigación "Formas de educación alternativa en el siglo XXI".

Entrevista 2. Jimena, educadora responsable de EnRonda. Entrevistada por Moira Alquezar en Mayo 2016. Proyecto de Investigación "Formas alternativas de educación en el siglo XXI".

Entrevista 3. Ileana, educadora responsable de El Canto de Fuego. Entrevistada por Francisco Ramallo en agosto de 2017. Proyecto de Investigación "Formas alternativas de educación en el siglo XXI". 